\title{
Picosecond Photoluminescence Decay Time in Colloidal Nanocrystals: The Role of Intrinsic and Surface States
}

\author{
Giovanni Morello,* Marco Anni, ${ }^{\dagger}$ Pantaleo Davide Cozzoli, Liberato Manna, \\ Roberto Cingolani, and Milena De Giorgi
}

National Nanotechnology Laboratory (NNL) of CNR-INFM, Distretto tecnologico ISUFI, Università degli Studi di Lecce, Via per Arnesano 73100 Lecce, Italy

Received: April 10, 2007

\begin{abstract}
Picosecond time-resolved photoluminescence measurements were performed on $\mathrm{CdSe}$ core and $\mathrm{CdSe} / \mathrm{ZnS}$ core/shell colloidal quantum dots (QDs). Photoluminescence (PL) emission is observed to originate from intrinsic $\pm 1^{U}$ and $\pm 1^{L}$ bright states with lifetimes of 60 and $450 \mathrm{ps}$, respectively, and from a long living component with nanosecond lifetimes. The latter is attribuited to the emission from surface states (ss) approximately 16 and $13 \mathrm{meV}$ below the $\pm 1^{L}$ state for core and core/shell QDs, respectively. We show that in the temperature range between 15 and $70 \mathrm{~K}$ the three recombination processes compete and they are thermally populated through different pathways $\left( \pm 1^{L} \rightarrow \pm 1^{U}\right.$ and ss $\left.\rightarrow \pm 1^{L}\right)$.
\end{abstract}

\section{Introduction}

Colloidal semiconductor quantum dots (QDs) have attracted great interest due to their peculiar optical properties, which can be controlled by tailoring their size, shape, and composition. ${ }^{1}$ In the last years, surfactant-assisted synthesis techniques have been significantly refined, leading to a remarkable improvement in photoluminescence (PL) quantum yield (QY) at room temperature, ${ }^{2-4}$ thus making such QDs suitable for various technologically relevant applications, such as light emitting diodes,${ }^{5,6}$ photovoltaic cells, ${ }^{7}$ and lasers. ${ }^{8}$ In particular, great improvement in QY has been obtained by surface passivation with a shell of a higher band gap semiconductor material, such that a type-I band alignment is achieved. ${ }^{9}$ In this regard, an understanding of the dependence of the temporal evolution of the radiative and nonradiative processes on the QD structure is therefore fundamental to optimize the nanostructure PL QY. To this aim, several experiments have been focused on the study of the dynamics of charge carriers under optical excitation. First, femtosecond pump-probe investigations on colloidal II-VI QDs have revealed that carriers cool nonradiatively into the emissive states in a few hundreds of femtoseconds. ${ }^{10,11}$ Recently, it has been verified that ultrafast relaxation arises from Auger-like, electron-hole interaction. ${ }^{12,13}$ Radiative relaxation channels in CdSe QDs have been investigated by time-resolved photoluminescence (TRPL), typically on the nanosecond time scale. These experiments usually exhibit biexponential decay traces. ${ }^{14,15}$ The shortest lifetime is of the order of several nanoseconds, whereas the longest one is on the time scale of tens of nanoseconds. The origin of these processes is still a matter of debate. Wang et al. have attributed the longest lifetime to surface state emission, ${ }^{14}$ whereas nonexponential traces, in the same temporal range, ${ }^{16}$ have been explained in terms of superposition of bright and dark states and of incomplete surface passivation. Relaxation processes on the microsecond time scale have also been observed and associated to dark state emission. ${ }^{17}$ Califano

* To whom correspondence should be addressed. E-mail: giovanni.morello@unile.it.

† Also with: Dipartimento di Ingegneria dell'Innovazione, Universitá degli studi di Lecce, Via per Arnesano 73100 Lecce, Italy. et al. ${ }^{18}$ have shown that the microsecond decay time is actually due to dark-bright state emission induced by the presence of surface states, while the dark state has been predicted to have a millisecond lifetime. At the single dot level, TRPL on CdSe QDs has revealed nanosecond lifetimes ${ }^{19}$ (emission from darkbright states) as well as nonexponential decays ${ }^{17}$ arising from fluctuating nonradiative relaxation channels. ${ }^{20,21}$ Concerning the bright states in the fine structure of CdSe QDs, Wang et al. ${ }^{22}$ have investigated for the first time the role of the $\pm 1^{U}$ and $\pm 1^{L}$ states in the carrier relaxation at dark and surface defect states. In spite of the great number of works dealing with TRPL on colloidal QDs, the role of $\pm 1^{U}$ and $\pm 1^{L}$ bright intrinsic states in the presence of emitting surface states has been only postulated. ${ }^{23-25}$ Bawendi et al. ${ }^{23}$ have showed transient emission from CdSe QDs involving surface states. These authors distinguished both a short lifetime of the order of their time resolution ( $\sim 100$ ps) probably arising from intrinsic emission, and a temperature-dependent interplay between the band edge and surface states. Henneberger et al. ${ }^{24}$ have found similar transient behavior on the same time scale, stressing the long decay time of radiative surface state emission. Recently, de Mello Donegá et al. $^{25}$ have investigated the temperature dependence of the exciton lifetime in CdSe QDs, finding evidence for a fast component in the time trace in the low temperature "radiative regime", that could not be resolved within their system (temporal resolution of $700 \mathrm{ps}$ ). Such contribution was ascribed to rapid carrier thermalization from the bright to dark states. To clearly assess the role of the bright states in the emission process, PL temporal evolution on a shorter time scale is required.

Here, we have studied the role of the $\pm 1^{U}$ and $\pm 1^{L}$ intrinsic bright states ${ }^{26}$ and surface states in the emission process both in colloidal CdSe QDs and in CdSe/ZnS core/shell QDs, employing TRPL on the picosecond time scale. We show that three states contribute to the emission spectra with three different decay times $\left(t_{1} \simeq 60 \mathrm{ps}, t_{2} \simeq 450 \mathrm{ps}\right.$, and $\left.t_{3} \simeq 10 \mathrm{~ns}\right)$. The individual contribution of each of the three states to the PL was 


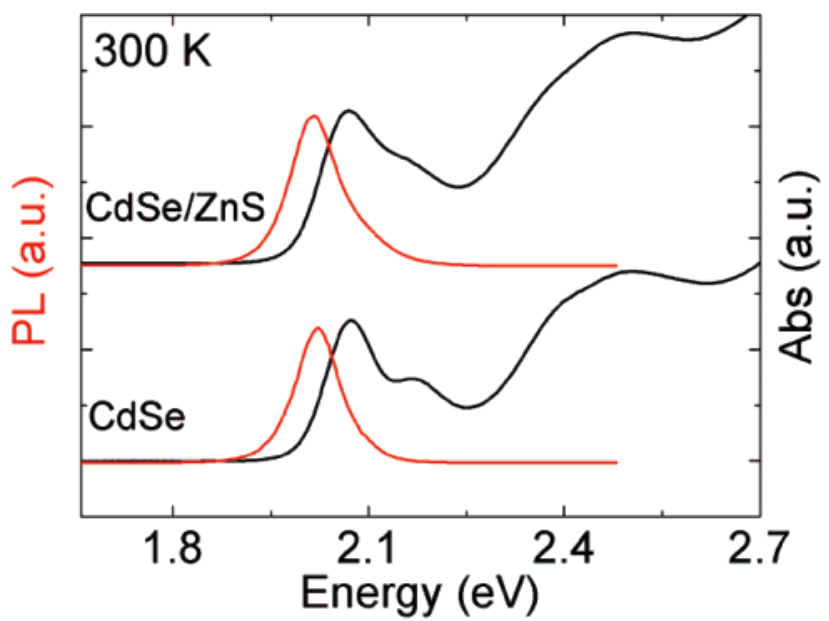

Figure 1. Absorption (black line) and PL spectra (red line) performed in solution for the two samples studied.

found to be temperature-dependent, due to the thermally induced filling of $\pm 1^{U}$ by $\pm 1^{L}$ and of both $\pm 1^{U}$ and $\pm 1^{L}$ by the surface state.

\section{Experimental Section}

A. Materials. Cadmium oxide ( $\mathrm{CdO}, 99.9 \%)$, diethylzinc $\left(\mathrm{Zn}\left(\mathrm{C}_{2} \mathrm{H}_{5}\right)_{2}, 1 \mathrm{M}\right.$ in hexane), bis(trimethylsilyl) sulfide (TMS, 99\%), selenium powders (Se, 99.999\%), tri-n-octylphosphine oxide (TOPO, 99\%), tri- $n$-butylphosphine $\left(\mathrm{P}\left(\mathrm{C}_{4} \mathrm{H}_{9}\right)_{3}\right.$ or TBP, $97 \%)$, and oleic acid $\left(\mathrm{C}_{17} \mathrm{H}_{33} \mathrm{CO}_{2} \mathrm{H}\right.$ or OLAC, $\left.90 \%\right)$ were purchased from Aldrich. $n$-Hexadecylamine $\left(\mathrm{NC}_{16} \mathrm{H}_{35}\right.$ or HDA, 99\%) was purchased from Fluka. Tri- $n$-octylphosphine $\left(\mathrm{P}\left(\mathrm{C}_{8} \mathrm{H}_{17}\right)_{3}\right.$ or TOP, $\left.97 \%\right)$ was purchased from Strem. All solvents used were anhydrous and were purchased from Aldrich and used without any further purification under nitrogen in a glove box.

B. Synthesis of CdSe QD Cores. All manipulations were performed using standard air-free techniques. CdSe QD cores were synthesized by using slightly modified literature protocols. ${ }^{9,27}$ Preliminarily, a stock Se solution was freshly prepared by dissolving $1.5 \mathrm{~g}$ of Se powders in $6 \mathrm{~g}$ of TBP under the $\mathrm{N}_{2}$ atmosphere of a glove box. CdSe nanocrystals (NCs) were synthesized by dissolving $\mathrm{CdO}(0.250 \mathrm{~g})$ with OLAC $(2.25 \mathrm{~g})$ in a flask at $\sim 200{ }^{\circ} \mathrm{C}$ under $\mathrm{N}_{2}$, followed by addition of TOPO $(5 \mathrm{~g})$ and $\mathrm{HDA}(5 \mathrm{~g})$ at room temperature. The resulting mixture was heated up to $270{ }^{\circ} \mathrm{C}$, and $2.5 \mathrm{~g}$ of the Se stock solution was added rapidly to the hot solution to induce QD nucleation. The temperature was kept at $250{ }^{\circ} \mathrm{C}$ during $\mathrm{NC}$ growth for 3-20 min, depending on the final QD size desired. The temporal evolution of QD growth was monitored by recording UV-vis absorption and photoluminescence spectra of small aliquots of the reaction mixture withdrawn with a syringe and then diluted with chloroform. The synthesis was halted by removing the heat source. The QDs were precipitated out of the surfactant mixture upon methanol addition, washed three times with methanol, and finally redissolved in chloroform. All these manipulations were carried out in the glove box.

C. Synthesis of CdSe/ZnS Core/Shell QDs. In a typical synthesis of CdSe/ZnS core/shell QDs, a calibrated amount of purified CdSe QDs prepared as described above was mixed with $5 \mathrm{~g}$ of TOPO, $1 \mathrm{~g}$ of TBP, and $2.5 \mathrm{~g}$ of HDA and heated to $220{ }^{\circ} \mathrm{C}$. The volume of the $\mathrm{Zn} / \mathrm{S}$ stock solution $(0.4 \mathrm{mmol} \mathrm{Zn}$ $\left(\mathrm{C}_{2} \mathrm{H}_{5}\right)_{2}$ and $0.5 \mathrm{mmol}$ TMS in $3 \mathrm{~mL}$ of TOP) necessary to obtain the desired shell thickness was calculated from the ratio between the core and shell volumes using the bulk lattice parameters of

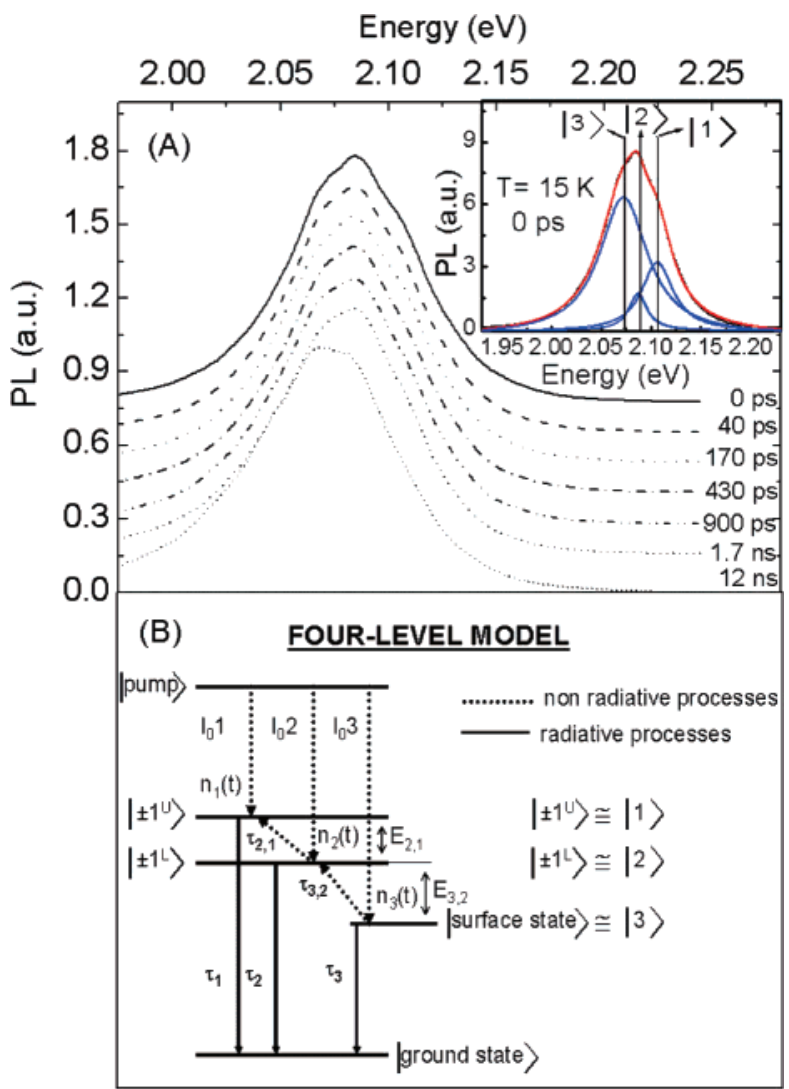

Figure 2. (A) Temporal evolution of the PL spectrum at $15 \mathrm{~K}$ for $\mathrm{CdSe} / \mathrm{ZnS}$ samples. The spectra consist of three bands evolving over time. The blue peak $(|1\rangle)$ evolution is the fastest, and the red one $(|3\rangle)$ is the slowest. After $1.7 \mathrm{~ns}$, a small red shift is observed because of the disappearence of feature $|1\rangle$, while after $12 \mathrm{~ns}$, peak $|3\rangle$ becomes dominant. All spectra were normalized to their peak value and vertically translated for clarity. The inset shows the best fit of the spectrum at $t$ $=0 \mathrm{ps}$ to a deconvolution of three Lorentzian curves. The extracted energy separations among the peaks are reported in the text. (B) Level scheme employed in the rate equation model to describe the emission spectra of core and core/shell QDs. After laser excitation, carriers relax nonradiatively to states $\left| \pm 1^{U}\right\rangle$ and $\left| \pm 1^{L}\right\rangle$ and to the surface state, from which they relax radiatively. The lifetime of states $\left| \pm 1^{U}\right\rangle$ and $\left| \pm 1^{L}\right\rangle$ is shorter than the intrinsic radiative decay time because of the fast carrier relaxation to the surface state, which emits with its intrinsic radiative decay time. Thermally induced population and depopulation of the states with nonradiative lifetime are considered.

$\mathrm{CdSe}$ and $\mathrm{ZnS}$. This volume was then added dropwise to the vigorously stirred hot solution of CdSe QD cores. The temporal evolution of the $\mathrm{ZnS}$ shell growth was monitored by recording UV-vis absorption and photoluminescence spectra on aliquots of the synthesis mixture. Typically, a maximum of the luminescence intensity was observed for a $\mathrm{ZnS}$ shell as thick as $\sim 1.5$ monolayers grown on 4.5-5 nm QD cores.

D. Transmission Electron Microscopy (TEM). The size of the QDs was checked by low-resolution TEM investigations. TEM images were recorded with a Jeol Jem 1011 microscope operating at an accelerating voltage of $100 \mathrm{kV}$. The samples for TEM were prepared by dropping a dilute chloroform solution of QDs onto carbon-coated copper grids and then allowing the solvent to evaporate.

E. Optical Absorption and Photoluminescence Spectroscopy on CdSe QD Solutions. UV-vis absorption spectra of the extracted nanocrystals were measured with a Cary 5 Varian UV-vis/near-IR spectrophotometer (see Figure 1). Photoluminescence (PL) emission spectra were measured with a Cary Eclipse spectrofluorimeter. PL quantum yields (QY) were 


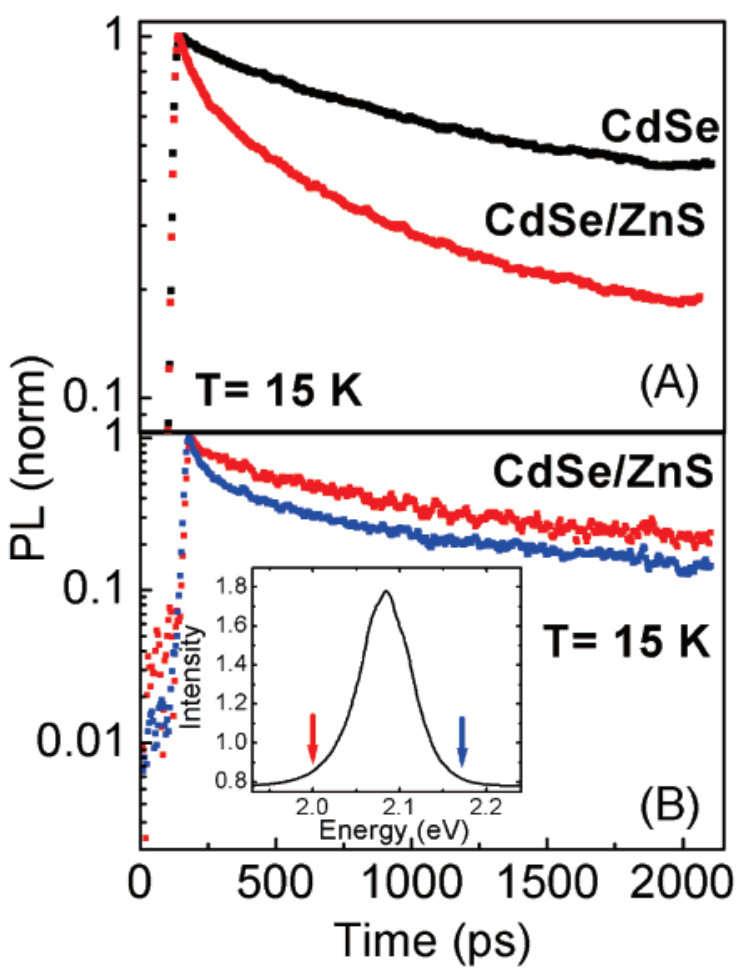

Figure 3. (A) Typical normalized time-resolved PL trace for CdSe QDs (black symbols) and CdSe/ZnS QDs with identical core size (red symbols) at $15 \mathrm{~K}$. (B) Comparison between the time traces on the blue (blue symbols) and red (red symbols) sides of the main PL signal (shown in the inset) at low temperature.

evaluated by comparing the QD emission intensity in chloroform solution with that of a reference dye (Rhodamine 6G) having the same optical density in ethanol solution. The measured QY of the core/shell QDs is 40\%, whereas the starting QD cores have a QY of $10 \%$.

F. Time-Resolved Photoluminescence Measurements. The QDs were deposited by drop casting a chloroform solution on $\mathrm{Si}-\mathrm{SiO}_{2}$ substrates, and then they were placed under vacuum in a closed cycle He cryostat. For each sample, we performed TRPL measurements in the temperature range of $15-300 \mathrm{~K}$, in steps of $10 \mathrm{~K}$. The samples were excited by the $80 \mathrm{fs}$ pulse of the second harmonic (397 nm) of a Ti:sapphire laser (repetition rate of $80 \mathrm{MHz}$ ). The $\mathrm{PL}$ signal was dispersed by a spectrograph (0.35 m focal length) and detected by a streak camera. The time resolution of the system was $\sim 12$ ps. All measurements were performed at low excitation intensity, corresponding to a photon fluence $\phi_{\mathrm{p}} \approx 7 \times 10^{11}$ photons $\mathrm{cm}^{-2} /$ pulse. By considering an absorption cross section $\sigma_{\mathrm{ab}}$ of $\sim 3 \times$ $10^{-15} \mathrm{~cm}^{2},{ }^{28}$ we estimated an average number, $N$, of excited carriers per $\operatorname{dot} N=\phi_{\mathrm{p}} \sigma_{\mathrm{ab}} \approx 2 \times 10^{-3}$. Such a small value allows us to avoid multicarrier scattering. ${ }^{29}$

\section{Results and Discussion}

For the purposes of the present discussion, two representative samples will be comparatively examined in detail, namely CdSe QDs with a mean size of $4.5 \mathrm{~nm}$ and therefrom synthesized $\mathrm{CdSe} / \mathrm{ZnS}$ core/shell QDs with a mean shell thickness of $\sim 1.5$ $\mathrm{ZnS}$ monolayers. Figure 1 displays the relevant optical absorp- tion and PL emission features of the selected nanocrystals, which satisfactorily reproduce literature data. ${ }^{9,27}$ Figure $2 \mathrm{~A}$ shows the temporal evolution of $\mathrm{CdSe} / \mathrm{ZnS}$ QD PL spectra at $15 \mathrm{~K}$ for different delay times, after the pump pulse. The $t=0 \mathrm{ps}$ spectrum corresponds to the maximum intensity time. The inset of Figure 2A shows a best fit of the spectrum to a superposition of three Lorentzian curves. The PL spectrum at $t=0 \mathrm{ps}$ peaks at around $2.084 \mathrm{eV}$ (this feature has been labeled as peak $|2\rangle$ ), with a low energy shoulder (peak $|3\rangle$ ) around $2.071 \mathrm{eV}$ and a high energy shoulder around $2.105 \mathrm{eV}(|1\rangle)$. The three trasitions show different relaxation dynamics. Peak $|1\rangle$ disappears almost completely in about $170 \mathrm{ps}$, whereas peak $|2\rangle$ shows a faster relaxation than peak $|3\rangle$ does. At $t=12 \mathrm{~ns}$, the PL spectrum is clearly red-shifted with respect to the one at $t=0 \mathrm{ps}$, and it is centered at around peak $|3\rangle$. Such a time evolution suggests that three emitting states, each with different relaxation times, should actually contribute to the PL of these quantum dots. In Figure $2 \mathrm{~B}$, we describe the system by a four-level model. The association of the three emitting states with two intrinsic states $\left(\left| \pm 1^{U}\right\rangle\right.$ and $\left.\left| \pm 1^{L}\right\rangle\right)$ and with one surface state will be discussed later. The energy separations extracted by the Lorentzian fit of the transient spectra (inset of Figure $2 \mathrm{~A}$ ) are $E_{2,1}=21 \mathrm{meV}$ and $E_{3,2}=13 \mathrm{meV}$ for $|2\rangle \rightarrow|1\rangle$ and $|3\rangle \rightarrow|2\rangle$, respectively. A similar behavior is observed for the CdSe core sample, although with energy separations of $21 \mathrm{meV}$ for $E_{2,1}$ and $16 \mathrm{meV}$ for $E_{3,2}$.

Figure 3A shows the normalized PL relaxation dynamics for the core and core/shell samples at $15 \mathrm{~K}$. A nonexponential decay can be observed for both samples. The PL time decay is well reproduced by a triexponential decay function at all temperatures and for both samples:

$$
I(t)=A_{1} \mathrm{e}^{-\left(t-t_{0}\right) / t_{1}}+A_{2} \mathrm{e}^{-\left(t-t_{0}\right) / t_{2}}+A_{3} \mathrm{e}^{-\left(t-t_{0}\right) / t_{3}}
$$

where $t_{0}$ is the delay at which $I(t)$ is maximum, $t_{1}, t_{2}$, and $t_{3}$ are the time constants, and $A_{1}, A_{2}$, and $A_{3}$ are the weights of each process. The best fit values of $t_{i}$ and $A_{i}(i=1-3)$ at $15 \mathrm{~K}$ are given in Table 1 for both samples. The still high value of $\mathrm{A}_{3}$ in the core/shell sample indicates that the $\mathrm{ZnS}$ shell does not passivate all the states present on the surface of the core sample.

It is worth noting that the experimental data can be reproduced, in the measured temporal range, neither by a simpler decay function (such as a biexponential decay) nor by the Williams-Watts function (stretched exponential). ${ }^{30}$ The latter should more appropriately be used only if the PL decay of the emitter ${ }^{16,31}$ is characterized by a quasi-continuous distribution of lifetimes. ${ }^{32}$ As opposed, if the decay can be ultimately traced by a finite number of exponentials, ${ }^{35}$ the time trace cannot be well fitted by a stretched function. ${ }^{33}$ Moreover, the nonexponential behavior can be neither due to Auger recombination, ${ }^{34}$ as the experiment was performed in a low excitation regime, nor due to energy transfer, ${ }^{35}$ since similar relaxation dynamics were also obtained in solution, where the average interparticle distance is too large to allow for efficient Förster resonant energy transfer (FRET). We can also exclude that the three spectral features could be due to emission from QD families of different mean size because the respective measured lifetimes are considerably different. We therefore ascribe the three decay times to the three transitions observed in the temporal evolution

TABLE 1: Best Fit Values of $t_{1}, t_{2}$, and $t_{3}$ and of the Relative Weights $A_{1}, A_{2}$, and $A_{3}$ for the Two Samples at $15 \mathrm{~K}$

\begin{tabular}{lcccccc}
\hline sample & $t_{1}(\mathrm{ps})$ & $t_{2}(\mathrm{ps})$ & $t_{3}(\mathrm{~ns})$ & $A_{1}$ & $A_{2}$ & $A_{3}$ \\
\hline CdSe & $62 \pm 4$ & $490 \pm 11$ & $10 \pm 1$ & $0.034 \pm 0.001$ & $0.29 \pm 0.03$ & $0.67 \pm 0.02$ \\
CdSe/ZnS & $61 \pm 1$ & $450 \pm 10$ & $9.5 \pm 0.7$ & $0.199 \pm 0.003$ & $0.474 \pm 0.002$ & $0.326 \pm 0.005$
\end{tabular}




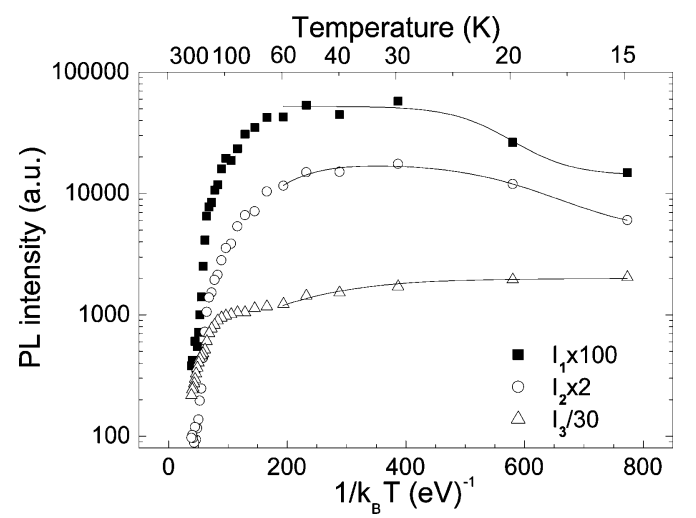

Figure 4. PL intensity of the three states as a function of temperature for $\mathrm{CdSe} / \mathrm{ZnS}$ QDs. The PL thermal increase for $I_{1}$ and $I_{2}$ at low temperatures is evident. The continuous lines are the fit curves to eqs $3-5$ for $I_{1}, I_{2}$, and $I_{3}$, respectively.

of the PL spectra shown in Figure 2A. This conclusion is confirmed by the energy dependence of the PL relaxation dynamics. Figure $3 \mathrm{~B}$ shows the normalized time traces at two different energies measured on the blue $(2.17 \mathrm{eV})$ and red $(2$ $\mathrm{eV}$ ) tails of the PL spectrum. The relaxation dynamics in the blue side shows an increased contribution of the fastest decay process as opposed to the red side dynamics, which is governed by the slowest process. The energy separation of $21 \mathrm{meV}$ between peaks $|1\rangle$ and $|2\rangle$, which does not change in the core and core/shell samples, together with the correspondent values of the measured decay times $t_{1}$ and $t_{2}$ (reported in Table 1), ${ }^{22}$ suggests that peaks $|1\rangle$ and $|2\rangle$ are due to the intrinsic states $\left| \pm 1^{U}\right\rangle$ and $\left| \pm 1^{L}\right\rangle$ (Figure 2B), that is, the lowest bright levels of the fine structure of the $1 S_{3 / 2} \rightarrow 1 S_{e}$ exciton state in spherical CdSe QDs. ${ }^{26}$ In fact, the theoretically predicted splitting between $\left| \pm 1^{U}\right\rangle$ and $\left| \pm 1^{L}\right\rangle$ is of $\sim 20 \mathrm{meV}$ in the large $1.7-5 \mathrm{~nm}$ size range. ${ }^{36}$ Concerning the state $|3\rangle$, its lifetime of several nanoseconds, the different energy separation from the state $\left| \pm 1^{L}\right\rangle$ found for core and core/shell QDs, and its stronger intensity (see Table 1) in the core sample (which has only an organic surface passivation) all suggest that it should be due to an extrinsic state, most likely a surface state. The existence of such surface states is confirmed by recent up-conversion measurements on colloidal CdSe QDs revealing emission from surface states lying below the band edge at an energy value of the order of the $E_{3,2}$ value found in this work. ${ }^{37}$

To investigate thermal effects on the population of the three states, we analyzed the temperature dependence of the PL intensities $I_{1}, I_{2}$, and $I_{3}$ of states $|1\rangle,|2\rangle$, and $|3\rangle$, respectively. We found that $I_{1}$ and $I_{2}$ increase as the temperature increases up to $\sim 70 \mathrm{~K}$, whereas $I_{3}$ decreases steadily (see Figure 4 ).

The same behavior is observed for the CdSe core QDs. These results suggest that in the range of $15-70 \mathrm{~K}$ thermally induced population of states $|1\rangle$ and $|2\rangle$ occurs, fed by state $|3\rangle$. At higher temperatures, the overall PL intensity strongly decreases, indicating the occurrence of thermal activation of nonradiative relaxation channels, such as thermal escape induced by multiple longitudinal optical (LO) phonon absorption. ${ }^{28}$ To analyze the low-temperature processes involving states $|1\rangle,|2\rangle$, and $|3\rangle$, we considered a four-level system, as depicted in Figure $2 \mathrm{~B}$. We assume that, after laser excitation, carriers relax nonradiatively from a pumping state (see Figure 2B) by Auger relaxation via electron-hole scattering ${ }^{22,38}$ to states $\left| \pm 1^{U}\right\rangle$ and $\left| \pm 1^{L}\right\rangle$ and to the surface state. In this model, population and depopulation processes are taken into account, with the relative rates $1 / \tau_{2,1}$ and $1 / \tau_{3,2}$. The rate equation system for the populations of $\left| \pm 1^{U}\right\rangle$ and $\left| \pm 1^{L}\right\rangle$ and of the surface states is therefore the following:

$$
\left\{\begin{array}{l}
\frac{\mathrm{d} n_{3}(t)}{\mathrm{d} t}=n_{03} \delta(t)-\frac{n_{3}(t)}{\tau_{3,2}} \mathrm{e}^{-E_{3,2} / k_{\mathrm{B}} T}-\frac{n_{3}(t)}{\tau_{3}} \\
\frac{\mathrm{d} n_{2}(t)}{\mathrm{d} t}=n_{02} \delta(t)-\frac{n_{2}(t)}{\tau_{2,1}} \mathrm{e}^{-E_{2,1} / k_{\mathrm{B}} T}+\frac{n_{3}(t)}{\tau_{3,2}} \mathrm{e}^{-E_{3,2} / k_{\mathrm{B}} T}-\frac{n_{2}(t)}{\tau_{2}} \\
\frac{\mathrm{d} n_{1}(t)}{\mathrm{d} t}=n_{01} \delta(t)+\frac{n_{2}(t)}{\tau_{2,1}} \mathrm{e}^{-E_{2,1} / k_{\mathrm{B}} T}-\frac{n_{1}(t)}{\tau_{1}}
\end{array}\right.
$$

where $n_{01}, n_{02}$, and $n_{03}$ represent the respective initial population of the three states as generated by the laser pulse (the latter is treated as a $\delta$ function because the pump pulse is much shorter than all the processes considered here); $\tau_{1}, \tau_{2}$, and $\tau_{3}$ are the intrinsic radiative decay times of the states; $1 / \tau_{2,1}$ and $1 / \tau_{3,2}$ are the depletion rates of the state $\left| \pm 1^{L}\right\rangle$ and the surface state, respectively; and $E_{2,1}$ and $E_{3,2}$ are the energy separations among the three states as indicated in Figure 2B. By solving the system, we obtain the expressions for the time-dependent populations $n_{1}(t), n_{2}(t)$, and $n_{3}(t)$ of the states involved in the emission process. Thus, the PL integrated areas $I_{1}(T), I_{2}(T)$, and $I_{3}(T)$ are as follows:

$$
\begin{gathered}
I_{1}(T)=\int_{0}^{\infty} \frac{n_{1}(t)}{\tau_{1}} \mathrm{~d} t=I_{01}+\frac{\tau_{2}}{\tau_{2,1}} \mathrm{e}^{-E_{2,1} / k_{\mathrm{B}} T}\left(I_{02}+I_{03} \rho\right) \\
I_{2}(T)=\int_{0}^{\infty} \frac{n_{2}(t)}{\tau_{2}} \mathrm{~d} t=\frac{I_{02}+I_{03} \rho}{1+\frac{\tau_{2}}{\tau_{2,1}} \mathrm{e}^{-E_{2,1} / k_{\mathrm{B}} T}} \\
I_{3}(T)=\int_{0}^{\infty} \frac{n_{3}(t)}{\tau_{3}} \mathrm{~d} t=\frac{I_{03}}{1+\frac{\tau_{3}}{\tau_{3,2}} \mathrm{e}^{-E_{3,2} / k_{\mathrm{B}} T}}
\end{gathered}
$$

where

$$
\rho=\frac{\frac{\tau_{3}}{\tau_{3,2}} \mathrm{e}^{-E_{3,2} / k_{\mathrm{B}} T}}{1+\frac{\tau_{3}}{\tau_{3,2}} \mathrm{e}^{-E_{3,2} / k_{\mathrm{B}} T}}
$$

By fitting the experimental data of Figure 4 to eqs $3-5$, we obtain an overall good agreement with the best fit parameters $E_{2,1}=20 \pm 1 \mathrm{meV}$ for core and core/shell QDs and $E_{3,2}=12$ \pm 1 and $16.5 \pm 0.3 \mathrm{meV}$ for $\mathrm{CdSe} / \mathrm{ZnS}$ and $\mathrm{CdSe}$, respectively. These values are very similar to those of the respective energy splitting extracted from the deconvolution of the spectra in Figure 2A. This analysis confirms that the emission of the samples is due to the superposition of the PL arising from three states, with an energetic separation compatible to the one previously assigned to the states $\left| \pm 1^{U}\right\rangle$ and $\left| \pm 1^{L}\right\rangle$ and to the surface state.

\section{Conclusions}

In summary, we studied the time evolution of the photoluminescence in colloidal $\mathrm{CdSe}$ and $\mathrm{CdSe} / \mathrm{ZnS}$ QDs on the picosecond time scale. In the first 2 ns, the temporal evolution of the spectra shows that the PL arises from three states with different time constants $\left(t_{1} \simeq 60 \mathrm{ps}, t_{2} \simeq 450 \mathrm{ps}\right.$, and $t_{3} \simeq 9-10$ ns). We show that the faster states (higher energy) can be assigned to the bright states $\left| \pm 1^{U}\right\rangle$ and $\left| \pm 1^{L}\right\rangle$ in the fine structure picture, while the origin of the third one is clearly nonintrinsic. The latter state, most likely a surface state, contributes to the 
greater part of the PL from the CdSe core sample, whereas its contribution is remarkably reduced in core/shell QDs, because of the better surface passivation produced by the inorganic $\mathrm{ZnS}$ layer.

Acknowledgment. We would like to thank Paolo Cazzato and Diego Mangiullo for valuable technical assistance. This work was supported by the European Projects SA-NANO (Contract No. 013698) and by the Italian Ministry of Research (Contract No. RBIN048TSE).

\section{References and Notes}

(1) Murray, C. B.; Norris, D. J.; Bawendi, M. G. J. Am. Chem. Soc. 1993, 115, 8706 .

(2) Qu, L.; Peng, X. J. Am. Chem. Soc. 2002, 124, 2049.

(3) Dabbousi, B. O.; Rodriguez-Viejo, J.; Mikulec, F. V.; Heine, J. R.; Mattoussi, H.; Ober, R.; Jensen, K. F.; Bawendi, M. G. J. Phys. Chem. B 1997, 101, 9463 .

(4) Reiss, P.; Bleuse, J.; Pron, A. Nano Lett. 2002, 2, 781. 354.

(5) Colvin, V. L.; Schlamp, M. C.; Alivisatos, A. P. Nature 1994, 370,

(6) Coe, S.; Woo, W. K.; Bawendi, M. G.; Bulovic, V. Nature 2002, 420,800 .

(7) Greenham, N. C.; Peng, X.; Alivisatos, A. P. Phys. Rev. B 1996, $54,17628$.

(8) Klimov, V. I.; Mikhailovsky, A. A.; Xu, S.; Malko, A.; Hollingworth, J. A.; Leatherdale, C. A.; Eisler, H.-J.; Bawendi, M. G. Science 2000, $290,314$.

(9) Talapin, D. V.; Rogach, A. L.; Kornowski, A.; Haase, M.; Weller, H. Nano Lett. 2001, 1, 207. 589.

(10) Mhamed, M. B.; Burda, C.; El-Sayed, M. A. Nano Lett. 2001, 1,

(11) Burda, C.; El-Sayed, M. A. Pure Appl. Chem. 2000, 72, 165.

(12) Efros, A. L.; Kharchenko, V. A.; Rosen, M. Solid State Commun. 1995, 93, 281.

(13) Klimov, V. I.; McBranch, D. W. Phys. Rev. Lett. 1998, 80, 4028.

(14) Wang, X.; Qu, L.; Zhang, J.; Peng, X.; Xiao, M. Nano Lett. 2003. 3,1103 .

(15) Javier, A.; Magana, D.; Jennings, T.; Strouse, G. F. Appl. Phys. Lett. 2003, 83, 1423.

(16) Schöps, O.; Le Thomas, N.; Woggon, U.; Artemyev, M. V. J. Phys. Chem. B 2006, 110, 2074.
(17) Crooker, S. A.; Barrik, T.; Hollingsworth, H. A.; Klimov, V. I. Appl. Phys. Lett. 2003, 82, 2793.

(18) Califano, M.; Franceschetti, A.; Zunger, A. Nano Lett. 2005, 5, 2360 .

(19) Labeau, O.; Tamarat, P.; Lounis, B. Phys. Rev. Lett. 2003, 90, 257404.

(20) Schlegel, G.; Bohnenberger, J.; Potapova, I.; Mews, A. Phys. Rev. Lett. 2002, 88, 137401 .

(21) Fisher, B. R.; Eisler, H. J.; Stott, N. E.; Bawendi, M. G. J. Phys. Chem. B 2004, 108, 143.

(22) Wang, H.; de Mello Donegá, C.; Meijerink, A.; Glasbeek, M. J. Phys. Chem. B 2006, 110, 733 .

(23) Bawendi, M. G.; Carroll, P. J.; Wilson, W. L.; Brus, L. E. J. Chem. Phys. 1992, 96, 946.

(24) Jungnickel, V.; Henneberger, F. J. Lumin. 1996, 70, 238.

(25) de Mello Donegá, C.; Bode, M.; Meijerink, A. Phys. Rev. B 2006, 74,085320 .

(26) Efros, A. L.; Rosen, M.; Kuno, M.; Nirmal, M.; Norris, D. J.; Bawendi, M. Phys. Rev. B 1996, 54, 4843.

(27) Qu, L.; Peng, A.; Peng, X. Nano Lett. 2001, 1, 333.

(28) Valerini, D.; Cretí, A.; Lomascolo, M.; Manna, L.; Cingolani, R.; Anni, M. Phys. Rev. B 2005, 71, 235409.

(29) Klimov, V. I.; McBranch, D. W.; Leatherdale, C. A.; Bawendi, M. G. Phys. Rev. B 1999, 60, 13740 .

(30) Williams, G.; Watts, D. C. Trans. Faraday Soc. 1970, 66, 80.

(31) Jones, M.; Nedeljkovic, J.; Ellingson, R. J.; Nozik, A. J.; Rumbles, G. J. Phys. Chem. B 2003, 107, 11346.

(32) Lindsey, C. P.; Patterson, G. D. J. Chem. Phys. 1980, 73, 3348; 2003, 107, 13782.

(33) Piechal, B.; Suffczyński, J.; Trajnerowicz, A.; Goryca, M.; Kossacki, P.; Golnik, A.; Gaj, J. A. Acta Phys. Pol., A 2005, $108,831$.

(34) Lomascolo, M.; Cretí, A.; Leo, G.; Vasanelli, L.; Manna, L. Appl. Phys. Lett. 2003, 82, 418.

(35) Achermann, M.; Petruska, M. A.; Crooker, S. A.; Klimov, V. I. J. Phys. Chem. B 2003, 107, 13782.

(36) The electronic structure of the first excited state in core/shell QDs is mainly determined by the core (apart from a slight red shift of the optical transitions because of the weaker carrier confinement with respect the CdSe core QDs), so the $\left| \pm 1^{U}\right\rangle-\left| \pm 1^{L}\right\rangle$ splitting is comparable in both samples.

(37) Chen, W.; Joly, A. G.; McCready, D. E. J. Chem. Phys. 2005, 122, 224708

(38) Klimov, V. I. J. Phys. Chem. B 2000, 104, 6112. 